\title{
Optical scattering simulation of ice particles with surface roughness modeled using the Edwards-Wilkinson equation
}

\section{Jianing Zhang ${ }^{1}$, Lei Bi ${ }^{1}$, Jianping Liu ${ }^{1}$}

\author{
R. Lee Panetta ${ }^{1 *}$, Ping Yang ${ }^{1}$, George W. Kattawar ${ }^{2}$
}

1. Department of Atmospheric Sciences, Texas A\&M University, College Station, TX

2. Department of Physics and Astronomy, Texas A\&M University, College Station, TX

\section{For publication in the}

Journal of Quantitative Spectroscopy and Radiative Transfer

Corresponding author address: R. Lee Panetta, Department of Atmospheric Sciences, Texas A\&M University, College Station, TX 77845. Email: panetta@tamu.edu 


\begin{abstract}
Constructing an appropriate particle morphology model is essential for realistic simulation of optical properties of atmospheric particles. This paper presents a model for generating surface roughness based on a combination of methods from discrete differential geometry combined with a stochastic partial differential equation for surface evolution introduced by Edwards and Wilkinson. Scattering of light by roughened particles is simulated using the Invariant Imbedding T-Matrix (II-TM) method. The effects of surface roughness on the single-scattering properties, namely, the phase matrix, asymmetry factor, and extinction efficiency, are investigated for a single wavelength in the visible range and for a range of size parameters up to $\mathrm{x}=50$. Three different smooth shapes are considered: spherical, spheroidal, and hexagonal, the latter two in just the "compact particle" case of unit aspect ratio. It is shown that roughness has negligible effects on the optical scattering properties for size parameters less than 20. For size parameters ranging from 20 to 50 , the phase matrix elements are more sensitive to the surface roughness than are two important integral optical properties, the extinction efficiency and asymmetry factor. As has been seen in studies using other forms of roughening, the phase function is progressively smoothed as roughness increases. The effects on extinction efficiency are to increase it, and on asymmetry factor is to decrease it. Each of these effects is relatively modest in the size range considered, but the trend of results suggests that greater effects will be seen for size parameters larger than ones considered here.
\end{abstract}


Keywords: Particle surface roughness, light scattering, Edwards-Wilkinson equation, discrete differential geometry 


\section{INTRODUCTION}

Particle shape irregularity is one of the most important factors that determine the single-scattering properties of atmospheric particles such as ice crystals in cirrus clouds [1-5]. Improvements in our understanding of the effects of surface roughness are of particular value for applications in remote sensing [6-8]. In the literature, the effects of particle overall shape on the optical properties have in the past drawn much more attention than effects associated with surface roughness [9-14] although there is an increasing awareness of the importance of particle surface roughness [6]. We believe that to gain better understanding of potential effects of surface roughness in optical modeling, it is necessary to take some account of microphysical processes involved in atmospheric particle formation and evolution [6-8].

The dynamics of ice particle surface formation involve several complicated physical processes, for instance deposition, evaporation, coalescence and collision [15]. For large ice particles, modeling of surface microphysics has attained some success [16]. In [10], surface roughness was generated through superposition of random waves on 2-D patches, and these 2-D roughened patches were computationally glued together to form a roughened hexagonal prism. In [19], on the other hand, an approach using a "pseudorandom surface roughness" was used to model a spherical roughened particle. In this approach, Gaussian random perturbations were imposed independently at different points on a spherical surface. A weakness in this approach is the lack of any spatial correlation between points on the roughened surface, resulting in a model whose appearance can be substantially different from surfaces observed on naturally occurring ice particles. In fact, the surface roughness generated by the pseudo-random surface model has no basis in the 
physics of surface formation, and the resultant surface roughness may consequently not be physically meaningful. From the physical point of view it is more desirable to construct a model based on physical processes relevant to surface formation. An attractive approach that has been developed in studies of the dynamics of surface growth involves a phenomenological model presented as a stochastic partial differential equation (SPDE) and has been used in scattering studies for plane surfaces or spheres [17, 18, 20].

In this study we extend the SPDE approach, using it to generate surface roughness for more general geometric figures and then simulate the effect that the roughness has on the optical properties of atmospheric ice particles. It is a major challenge, however, to analytically solve a SPDE in general geometry and recourse is made to numerical simulation. Then it becomes a task of approximating solutions to the SPDE using a formulation of the equation appropriate to a discrete mesh. The recently developed Discrete Differential Geometry (DDG) method [21] retains key geometric properties of the continuous model and has been applied in such geometric studies as surface parameterization and re-meshing. In this work, we apply the approach to a wider class of particle shapes than considered in previous scattering studies.

Once a model particle has been constructed, we conduct the scattering simulations using the Invariant Imbedding T-Matrix (II-TM) method presented in Bi et al. [22, 23], a method applicable to particles with arbitrary shapes. The invariant imbedding algorithm was first introduced by [24], in which the T-matrix was derived using a volume integral equation formulation. However, the assumption [24] adopted for deriving the invariant imbedding equations has proved to be unnecessary, as discussed in [22]. The T-matrix method, which assumes linearity of the system (linear permittivity/permeability and 
Maxwell's equations), can be derived in other frameworks than that of [24], such as the boundary element method or discrete dipole approximation method [25].

The rest of this paper proceeds as follows. Section II first places the EdwardsWilkinson surface growth model in the context of a more general one, the Kardar-ParisiZhang model and outlines the physical effects being modeled. Then it outlines a stochastic discrete differential geometry approach for solving stochastic partial differential equations on an arbitrary shape. Section III presents numerical results and discusses the effects of particle surface roughness on the single-scattering properties. Conclusions are discussed in Section IV.

\section{MODEL}

\section{A. Surface Growth}

A natural ice particle or dust aerosol usually exhibits overall non-spherical particle geometry with small-scale surface roughness. A variety of different growth mechanisms have been proposed for modeling surface morphologies [15]. In this section, we briefly introduce a simple growth model and extend it to model the roughness of atmospheric particles.

For an ice particle, we assume that molecules evaporate or condense randomly on a surface and that the growth direction is along the local surface normal. A height function $h(\vec{x}, t)$ of points $\vec{x}$ on the surface can be defined as the deviation at time $t$ of the roughened surface from a point prescribed smooth surface, where the deviation is measured along the surface-normal direction. Thus $h=0$ corresponds to the smooth 
surface, and we assume that the modeling process starts from a smooth particle $h(\vec{x}, 0)=0$. The roughened particle then "grows" from this smooth particle as time increases. A model that has received considerable attention for the dynamics of surface roughness growth is the Kardar-Parisi-Zhang (KPZ) [27] equation:

$$
\frac{\partial h}{\partial t}=v \nabla^{2} h+\frac{\lambda}{2}(\nabla h)^{2}+\eta
$$

where $v \nabla^{2} h(\vec{x}, t)$ is a linear term, $\frac{\lambda}{2} \nabla h(\vec{x}, t) \cdot \nabla h(\vec{x}, t)$ is a quadratic non-linear term, and $\eta(\vec{x}, t)$ is a Gaussian white noise random variable in space and time that models random evaporation or condensation of molecules. Because of the presence of the random term $\eta(\vec{x}, t)$, Equation (1) is a stochastic partial differential equation. The statistical behavior of $h(\vec{x}, t)$ is determined by the first and second moments of $\eta(\vec{x}, t)$, which are taken to be

$$
\begin{aligned}
& <\eta(\vec{x}, t)>=0 \\
& <\eta(\vec{x}, t) \eta\left(\overrightarrow{x^{\prime}}, t^{\prime}\right)>=2 \sigma_{\eta}^{2} \delta\left(\vec{x}-\overrightarrow{x^{\prime}}\right) \delta\left(t-t^{\prime}\right)
\end{aligned}
$$

In Eqs. (2) and (3), $<\cdot>$ denotes the space-time expectation value operator and $\sigma_{\eta}$ is the standard deviation of the random variable $\eta$. During the growth process, the linear term $v \nabla^{2} h \quad(v>0)$ serves to smooth out the surface: $\boldsymbol{v}$ is a parameter related to the thermal equilibrium between the water vapor and ice phases. The nonlinear term $(\lambda / 2)(\nabla h)^{2}$ is associated with surface gradient [27], and is the first term in an expansion of surface curvature. The existence of the non-linear term renders the equation quite difficult to solve and a detailed discussion of the known properties of its solutions is beyond the scope of this paper. 
A simpler equation without the nonlinear term is obtained by setting $\lambda=0$, reducing the equation to the linear Edwards-Wilkinson [28] equation:

$$
\frac{\partial h}{\partial t}=\boldsymbol{v} \nabla^{2} h+\boldsymbol{\eta} .
$$

Equation (4) has the geometrical interpretation that the roughening process associated with random deposition $\eta$ is smoothed out by the diffusion term $v \nabla^{2} h$. It can be shown that as time progresses, the correlation length between the elevation at different points on the surface increases with time until the effects of a finite domain size are felt. Subsequent to that time a statistical-dynamic equilibrium may be approached, but we are here primarily interested in sampling surfaces in the early evolution from the smooth state, when the correlation length is small compared with the size of the particle. A study of sensitivity of optical scattering properties to the stopping time, and hence value of this length, will be the subject of future work.

\section{B. Discretization of the Stochastic Partial Differential Equation}

The equation (4) that describes surface growth is often presented in the context of roughening a two dimensional planar surface. Here we assume that an analogous equation may be used to model roughening of a two dimensional surface of a three dimensional ice particle, with the view that the growth then involves the ice particle surface growing along the surface normal under the combined effects of deposition and surface smoothing. As mentioned above, when it comes to numerical simulation, the continuous evolution equations must be somehow discretized. For a smooth surface embedded in three dimensions, it is relatively easy to define the normal direction-the unique direction orthogonal to all tangent vectors. But for a discretized surface, for 
instance one made up of a mesh of triangular planar surfaces, there is no unique normal direction for locations at corners or along edges: at such points the curvature is singular. This difficulty can be addressed by method used in discrete differential geometry [21]. The starting point in the method is a discretization of the continuous surface using a triangular mesh as shown in Figure 1. To solve the SPDE on a discrete surface, we must find an appropriate way to define the Laplacian operator for the diffusion term and find the surface normal at each vertex in the discrete space. We sketch an approach described in more detail in [21], here only describing the key features.

Each planar triangular element in the mesh has three vertices. The evolution equation (4) is re-formulated as an equation for the evolution of the displacement of the vertex along an outward normal from the smooth surface. Assuming an enumeration of vertices in the mesh and using first-order forward time-stepping, equation (4) has the discrete analogue at mesh vertex with index $\mathrm{j}$

$$
h_{j}^{i+1}=h_{j}^{i}+v \delta t\left(\nabla^{2} h\right)_{j}^{i}+\eta_{j}^{i} \delta t
$$

where $\mathrm{i}$ is an index for time and the $\eta_{j}^{\mathrm{i}}$ are chosen independently from a Gaussian distribution. The crucial step in the method is how the representation of the Laplacian term is calculated. This is done by the "cotan formula" commonly used in finite element methods. The formula is a sum of terms corresponding to all vertices joined by an edge of the mesh to the vertex $h_{j}$, at time level $\mathrm{i}$ :

$$
\left(\nabla^{2} h\right)_{j}^{i}=\frac{1}{2} \sum_{m}\left(\cot \alpha_{m}-\cot \beta_{m}\right)\left(h_{m}^{i}-h_{j}^{i}\right) .
$$

In this formula, $m$ is an index over other vertices sharing an edge with vertex $j$, and the 
angles $\alpha$ and $\beta$ are the angles opposite that edge in the corresponding triangular element. The correlation length, the distance at which the correlation of height at a vertex falls off by a factor of $1 / \mathrm{e}$, can be shown to be $\sqrt{2 n \delta t}$ with $\mathrm{n}$ the number of time steps. Thus, as mentioned above, it grows with time.

Using this method, a surface with uniformly distributed roughness can be defined on arbitrary geometric objects. Figure 2 shows examples of roughened spheres, spheroids and hexagonal ice particles. In the figure, smoothed $(\mathrm{t}=0)$ particles are shown in the left-most column, and the columns to the right show evolved surfaces at two later times.

\section{Height and Slope Statistics}

Before considering the scattering properties of roughened particles, we first illustrate some statistical properties of the surface roughness model when it is applied to a simple plane surface. The heights at surface points obey Gaussian distribution with a mean zero and standard deviation $w$. Following what is perhaps unfortunate but nevertheless common usage, we call $w$ the "width" of the surface height distribution. It can be shown that the distribution of local slopes is also normal [15]. The width and the distribution of slopes are key elements in statistical theories of roughness effects on scattering, for example in the "tilted facet" method of [1]. In that method, the slope distribution of a rough surface is assumed to follow a Gaussian:

$$
p(\nabla h)=\frac{1}{\pi \sigma^{2}} \exp \left(-|\nabla h|^{2} / \sigma^{2}\right)
$$


In this expression, the vector "slope function" $\nabla h=\left(\frac{\partial h}{\partial u}, \frac{\partial h}{\partial v}\right)$, where (u, v) are orthonormal coordinates in a local frame of a smooth particle. It is known that $|\nabla h|^{2}$ then obeys a Weibull distribution with $a=\sigma^{2}, \quad b=1$, with the probability density function for the 2-parameter Weibull distribution given by

$$
p(x)=\frac{b}{a}\left(\frac{x}{a}\right)^{b-1} \exp \left(-(x / a)^{b}\right) \quad x \geq 0 ; a, b>0 .
$$

In the tilted facet method, particle surface details are not considered, and in fact no single particle is actually constructed. The theory produces a far-field scattering pattern that is determined entirely by the surface slope distribution. A comparison between results from the tilted facet method and ones obtained by the exact pseudo-spectral time-domain model with the random wave surface model was conducted by Liu et al[10]. It is not our aim to repeat such a study with the current surface roughness model, but we do show the degree to which the model surface constructed using the Edwards-Wilkinson equation satisfies the statistical assumptions of the tilted facet model in the case of a simple planar surface.

To analyze the statistical parameters associated with a roughened surface, we fix the time of simulation. (Hence the correlation length is fixed accordingly.) Figure 1a shows the surface at a given time, with a distribution of surface-normal vectors as indicated by red arrows. Since the width $\mathrm{w}$ of the surface height distribution and the variance of the surface slope distribution are related to each other through the correlation length [15], we can test the response of slope to the change of surface height. To simplify the problem, $|\nabla h|^{2}$ is tested instead of the two individual slope function components. Figure 3 shows 
height and slope distributions with the estimated parameters. Figure 4 illustrates the scatter plot of $\sqrt{\hat{\sigma}^{2}}$, the sample standard deviation of the slopes as computed from the surface produced by our evolution model against $\hat{w}$, along with the regression line.

\section{NUMERICAL SIMULATIONS AND RESULTS}

We now turn to the simulation of scattering properties for our roughened particles. In the simulations producing the particles, we model the ice particles discretized using a triangular mesh with up to 10000 triangular elements. Three model ice particles (sphere, spheroid and hexagon) are chosen for comparison with their roughened counterparts. All numerical simulations are done with a computer code based on the II-TM, a parallelized code written in Fortran90 with multi-threading implemented using OpenMP. Twenty cores are used for each size parameter to speed up the calculation. A refractive index of 1.3 is used for simulating the scattering of visible light by the roughened ice crystals.

Figures 5-7 show contour plots of the reduced Mueller matrix $\left(\mathrm{P}_{11}, \mathrm{P}_{12} / \mathrm{P}_{11}\right.$, and $\mathrm{P}_{22} / \mathrm{P}_{11}$ ) for the roughened particles (spheres, spheroids, and hexagonal prisms). All the matrix elements are shown as functions of scattering angle (the horizontal axis) and the size parameter $\mathrm{x}=\mathrm{ka}$ (the vertical axis), defined using of the smooth particle geometry. For spheres, a is the radius. For the spheroid, a is the distance from its center to its pole along the symmetry axis. Letting $\mathrm{b}$ being the equatorial radius, the particle is chosen to have $b=a / 2$. For the hexagon, $a$ is the radius of a circle circumscribing the base, and the height $\mathrm{L}$ of the hexagon is chosen to be $2 \mathrm{a}$, making the hexagon have unit aspect ratio. The results shown are for "random orientations," i.e. they have been averaged over incident angles. 
The roughened spheres involve surface structure on a spherical particle shape. We expect that when the particle height distribution has values much smaller than the incident wavelength, the roughened particle will behave optically essentially like a smooth sphere; the roughened sphere will be "optically spherical." Thus, $\mathrm{P}_{22} / \mathrm{P}_{11}$ can be an indicator of the size parameter region where the roughened sphere is optically nonspherical, and hence where a spherical approximation to the roughened sphere may be inappropriate for determining the optical properties. The bottom panel of Figure 5 shows that $\mathrm{P}_{22} / \mathrm{P}_{11}$ deviates dramatically from unity at large scattering angles when the size parameter is larger than approximately 20 . We note that the average value of the height distribution increases with the size parameter, although the surface roughness pattern is invariant. The diagrams of $\mathrm{P}_{12} / \mathrm{P}_{11}$ shown in the middle panel have many local extrema, presumably due to optical interference [31]. Calculations not illustrated here show that an increase of $\sigma$ results in smoother patterns, especially for larger sizes. The physical mechanism leading to a smoother pattern may be the randomization of phases associated with scattered wavelets as well as the orientation averaging process. Particles clearly show more pronounced non-spherical features with the growth of roughness. The overall geometric similarity of shapes of roughened spheres and smooth spheres may explain the similarity in macroscopic patterns of $\mathrm{P}_{11}$ and $\mathrm{P}_{12} / \mathrm{P}_{11}$. Figure 6 shows the results for spheroids. Unlike the case of the roughened sphere, the non-sphericity effect for a roughened spheroid is apparent throughout the size parameter range considered above approximately $x=10$. However, the non-sphericity effects more likely stem from the overall non-spherical shape of the particles rather than from the surface roughness. A similar remark applies in the case of hexagonal ice particles. 
Surface roughness may play different roles for spherical and non-spherical particles. For roughened spheres, the surface roughness has both qualitative and quantitative effects, because the roughness induces non-sphericity. From the simulated results of spheroids and hexagonal particles, we find that overall non-spherical particle shape has the most effect on the particle depolarization ratio, although the surface roughness does produce additional variation. For example, the maximum value of depolarization ratio of the roughened spheres is much smaller than the corresponding maxima for roughened non-spherical particles.

As seen from Figures 6 and 7, the roughness effects for non-spherical particles do not change the general pattern in the two-dimenional space constructed from the size parameter and the scattering angle. However, the absolute values of phase matrix elements for smooth and roughened particles can be quite different when the size parameter is sufficiently large. For example, Figures 8-10 compare the phase matrices for the particles at a single size $(\mathrm{ka}=50)$. It is clear that roughness smoothes out the rapid oscillations and decreases the polarization and depolarization. For the roughened sphere, the roughness results in a side scattering enhancement for $\mathrm{P}_{11}$, while decreasing $\mathrm{P}_{22} / \mathrm{P}_{11}$ dramatically. For spheroids, the roughness also smoothes all phase matrix element oscillations, although those oscillations even without roughness are already much smaller in amplitude than in the spherical case. Roughness also leads to a significant backscattering enhancement, but the effect on $-\mathrm{P}_{12} / \mathrm{P}_{11}$ is smaller than in the spherical case, with the exception of a substantial decrease in a range of angles around $135^{\circ}$. For hexagons, roughness reduces the $22^{\circ}$ and $46^{\circ}$ halos, as observed a number of other studies. It also smoothes out the linear polarization and decreases the depolarization over the scattering angles. Other elements show similar response to roughness except $\mathrm{P}_{44} / \mathrm{P}_{11}$, 
which indicates a reverse in the trend of the curves. For a smooth ice crystal, such features as the peaks at $22^{\circ}$ and $46^{\circ}$ in the angular distribution of $\mathrm{P}_{11}$ that are due to symmetry features of the crystal geometry. Effects of disruptions of these symmetries are progressively averaged out as the height of the surface increases.

Figure 11 shows the extinction efficiency and the asymmetry factor for these model particles as functions of the size parameter ka. For the figure the extinction efficiency has been calculated by dividing the extinction cross section by $\pi x^{2}$, i.e. by the geometric cross section of a sphere whose radius is the particle size parameter. The figure shows that surface roughness has little distinguishable effect for any of the particle shapes for sizes in the Rayleigh range, and for all three basic particle shapes the effect in the larger size range is overall to slightly increase the extinction efficiency. For smooth spheres, the extinction efficiency curve has small-scale oscillations superposed on the larger scale oscillations. For roughened spheres, the small-scale oscillations disappear as the size parameter increases, presumably due to suppression of the electromagnetic surface waves. But roughness has negligible effect on the larger scale oscillations of the extinction curve, perhaps because the induced phase-delay difference associated with transmitted rays is small.

Turning to the asymmetry parameter, once again the effects of roughness only emerge as the size parameter grows. For spheres, which are strongly forward scattering, the effect of roughness becomes evident once the size parameter exceeds about 15 . The effect is to decrease the asymmetry parameter because of the increased scattering away from the forward directions. This effect can be seen clearly in Figure 8, which shows that the phase function of a roughened sphere is generally larger than that of a smooth sphere at 
scattering angles larger than $60^{\circ}$. Spheroids already have a degree of asymmetry even in the smooth case, and it therefore takes a larger size parameter (about $\mathrm{x}=25$, according to Figure 11) for the effects of roughness to become evident; again the effect is to decrease the asymmetry.

By comparing Figure 11 (middle and left plots), we see that the extinction efficiencies for smooth spheroids clearly differ from those of smooth spheres, and that the inclusion of surface roughness modifies only modestly the values in the size parameter range considered. The overall shape change (from sphere to spheroids) is much more effective than the surface roughness in changing the optical properties. Put differently, the overall particle shape is a first-order factor in determining the integrated optical properties, whereas roughness produces only second-order modifications of the integrated optical properties of smooth particles. Hexagonal particles (rightmost plots in Figure 11) barely show the reduction of asymmetry factor at larger size parameters in the range considered that is seen in the sphere and spheroid cases, apparently because the first order effect of basic shape change already results in a large reduction in asymmetry parameter, and it is hard to see much effect until about $x=35$. There is a suggestion in the figure that if the size parameter range were widened to include size parameters larger than 50 , the same kind of asymmetry parameter lowering would become more prominent for hexagonal crystals as was seen for spheres and spheroids at smaller values of the size parameter.

\section{CONCLUSIONS}

We have investigated effects of surface roughness on ice particle scattering properties using a new statistical model. In our model, surface roughness was defined in terms of a height function that satisfies a discretized version of the Edwards-Wilkinson 
stochastic partial differential equation. The model makes possible a uniform construction of roughness on particle surfaces that include corners and edges. We simulated the light scattering by the model particles using the Invariant Imbedding T-Matrix method, a rigorous method that obtains the optical properties of arbitrarily shaped particles from solutions to Maxwell's equations. We investigated roughness effects on the optical properties of spheres, spheroids and hexagonal ice particles. The effects on the phase matrix become clear only when the size parameter is larger than 20 . In particular, the salient features associated with symmetries of smooth particles (rainbow and halos) disappear with increase of the degree of roughness, presumably because the wave paths leading to these optical phenomena are distorted by the roughened surfaces. Roughness effects on the integrated optical properties (extinction efficiency and asymmetry factor) are relatively modest when the size parameter is smaller than 50 . The effect on the extinction efficiency is generally to raise it, and the effect emerges at smaller size parameters for hexagons than for spheroids or spheres. On the other hand, the effect on asymmetry parameter, which is to decrease it, emerges first at smaller size parameters for spheres than for the less inherently symmetric spheroidal and hexagonal crystal. The actual values of size parameters at which each shape first shows sensitivity to roughness depends of course on wavelength, as well as the particular values of a number of other parameters held fixed in this study like incident wavelength, aspect ratio, surface slope variance.

The principle advantage of the present model presented here is that it is based on a phenomenological model of growth that has a clear physical justification, a model that has proven to be useful in other physical contexts. One feature of the model that we have postponed for future study is the sensitivity of scattering properties to the correlation length of the surface roughness, a property that dynamically evolves in the model. Other 
subjects for future study, aside from the sensitivities just mentioned, are the application of the present model in remote sensing studies and radiative transfer simulations, as well as consideration of surfaces constructed using the more general nonlinear Kardar-ParisiZhang equation.

\section{ACKNOWLEDGMENTS}

Numerical simulations were carried on the supercomputing platform of Texas A\&M University. The authors acknowledge the facility staff for their assistance. Partial support was obtained from NSF grants AGS-1338440 and OCE 1130906.

[1] P. Yang and K. N. Liou, Single-scattering properties of complex ice crystals in terrestrial atmosphere, Contr. Atmos. Phys. 71, 223-248(1998).

[2] V. Shcherbakov, J. F. Gayet, B. Braker, P. Lawson, Light scattering by single natural ice crystals, J. Atmos. Sci. 63(5),1513-1525(2006).

[3] A. Ehrlich, M. Wendisch, E. Bierwirth, A. Herber, A. Schwarzenbock, Ice crystal shape ef- fects on solar radiative properties of Arctic mixed-phase clouds-Dependence on microphysical properties, Atmos. Res.88, 266-276 (2008).

[4] A. J. Baran, A review of the light scattering properties of cirrus, J. Quant. Spectrosc. Radiat. Transf. 110, 1239-1260 (2009).

[5] A. J. Baran, From the single-scattering properties of ice crystals to climate prediction: A way forward, Atmos. Res. 112, 45-69 (2012).

[6] B. A. Baum, P. Yang, Y. -X. Hu, and Q. Feng, The impact of ice particle roughness on the scattering phase matrix, J. Quant. Spectrosc. Radiat. Transf. 111, 2534-2549 (2010).

[7] W. C. Pfalzgraff, R. M. Hulscher, and S. P. Neshyba, Scanning electron microscopy and molecular dynamics of surfaces of growing and ablating hexagonal ice crystals, Atmos. Chem. Phys. 10 2927-2935 (2010).

[8] Z. Ulanowski, P. H. Kaye, E. Hirst, R. S. Greenaway, R. J. Cotton, E. Hesse, and C. T. Collier Incidence of rough and irregular atmospheric ice particles from Small Ice 
Detertor 3 measurements, Atmos. Chem. Phys., 14, 1649-1662(2014).

[9] S. Schröoder, A. Duparr'e , L. Coriand, A. Tünnermann, D. H. Penalver, and J. E. Harvey Modeling of light scattering in different regimes of surface roughness, Opt. Express 19, 9820- 9835 (2011).

[10] C. Liu, R. L. Panetta, and P. Yang, The effects of surface roughness on the scattering properties of hexagonal columns with sizes from the Rayleigh to the geometric optics regimes, J. Quant. Spectrosc. Radiat. Transf. 129, 169-185 (2013).

[11] T. Nousiainen, K. Muinonen, Surface-roughness effects on single- scattering properties of wavelength-scale particles, J. Quant. Spectrosc. Radiat. Transf. 106, 389397(2007).

[12] M. Kahnert, T. Nousiainen, P. Mauno, On the impact of non-sphericity and smallscale surface roughness on the optical properties of hematite aerosols, J. Quant.

Spectrosc. Radiat. Transf. 63, 1513-1525(2006).

[13] M. Kahnert, T. Rother, Modeling optical properties of particles with small-scale surface rough- ness: combination of group theory with a perturbation approach, Opt. Express 1911138-11151(2011).

[14] M. Kahnert,T. Nousiainen, M. A. Thomas, J. Tyynel, Light scattering by particles with small- scale surface roughness: Comparison of four classes of model geometries, $\mathrm{J}$ Quant Spectrosc Radiat Transfer 113, 2356-2367(2012).

[15] Y-P Zhao, G. Wang, T-M Lu Characterization of Amorphous and Crystalline Rough Surface- Principles and Applications, (Academic Press, San Diego, CA, 2001).

[16] J. M. Edwards, S. Havemann, J. C. Thelen, A. J. Baran, A new parameterization for the radiative properties of ice crystals: comparison with existing schemes and impact in a GCM, Atmos. Res. 83, 19-34(2007).

[17] A. A. Maradudin Light Scattering and Nanoscale Surface Roughness, (Springer, New York, 2007).

[18] K. Muinonen, T. Nousiainen, P. Fast, K. Lumme, J. Peltoniemi, Light scattering by Gaussian random particles: Ray optics approximation, J. Quant. Spectrosc. Radiat.

Transf. 55, 577-601(1996).

[19] C. Li, G. W. Kattawar, and P. Yang, Effects of surface roughness on light scattering by small particles, J. Quant. Spectrosc. Radiat. Transf. 89, 123-131 (2004).

[20] T. Nousiainen, G. M. McFarquhar, Light scattering by quasi-spherical ice crystals, J. Atmos. Sci. 61,2229-2248(2004).

[21] K. Crane, F. de Goes, M. Desbrun, P. Schröoder, Digital Geometry Processing with Discrete Exterior Calculus, ACM SIGGRAPH 2013 courses, SIGGRAPH '13 (56), 899 
(ACM, New York, NY)(2013).

[22] L. Bi, P. Yang, G. W. Kattawar, M. I. Mishchenko, Efficient implementation of the invariant imbedding T-matrix method and the separation of variables method applied to large nonspher- ical inhomogeneous particles, J Quant Spectrosc Radiat Transf 116, 169183(2013).

[23] L. Bi, P. Yang, Accurate simulation of the optical properties of atmospheric ice crystals with the invariant imbedding T-matrix method, J. Quant. Spectrosc. Radiat. Transf. 138, 17-35(2014).

[24] B. R. Johnson Invariant imbedding T-matrix approach to electro- magnetic scattering, Appl. Opt. 27, 4861-4873(1988).

[25] M. I. Mishchenko, G. Videen, N. G. Khlebtsov, T. Wriedt, N. T. Zakharova, Comprehensive T-matrix reference database: A 2006-07 update, J. Quant. Spectrosc. Radiat. Transf. 109, 1447-1460(2008).

[26] D. W. Mackowski, Discrete dipole moment method for computing the T matrix for nonspherical particles, J. Opt. Soc. Am. A 19, 881-893 (2002).

[27] M. Kardar, G. Parisi, and Y.-C. Zhang, Dynamical scaling of growing interfaces, Phys. Rev. Lett. 56, 899 (1986).

[28] S. F. Edwards and D. R. Wilkinson, The surface statistics of a granular aggregate, Proceedings of the Royal Society (London) A 381, 17 (1982).

[29] M. Kru ger, G.Bimonte, T. Emig, and M. Kardar, Trace formulas for nonequilibrium Casimir interactions, heat radiation, and heat transfer Phys. Rev. B. 86, 115423(2012).

[30] P. C. Waterman, Symmetry, Unitarity, and geometry in electromagnetic scattering, Phys Rev D 3 825-839(1971).

[31] M. I., Mishchenko, and L.D. Travis, T-matrix computations of light scattering by large spheroidal particles, Opt. Comm. 109, 16-21(1994). 


\section{Figures:}

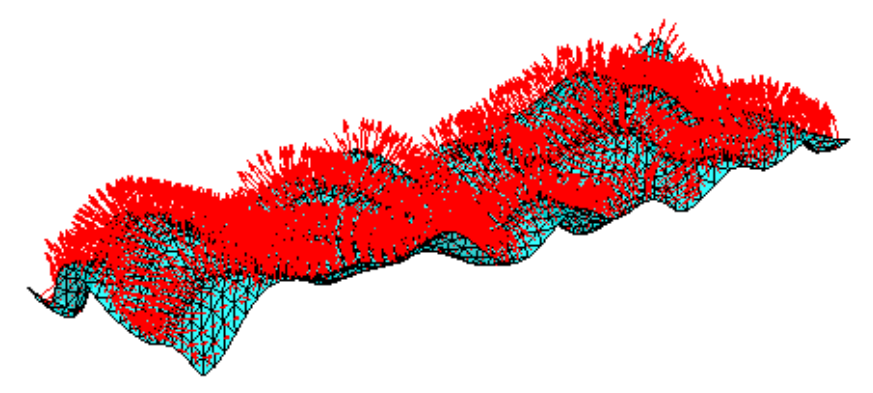

(a)

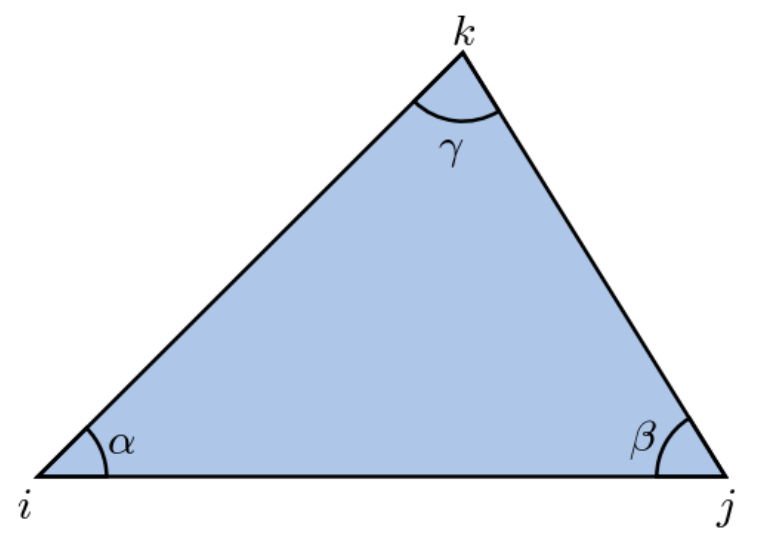

(b)

Figure 1. (a) Triangular element mesh plot with surface normal (red). (b) Typical triangular element of the mesh. 

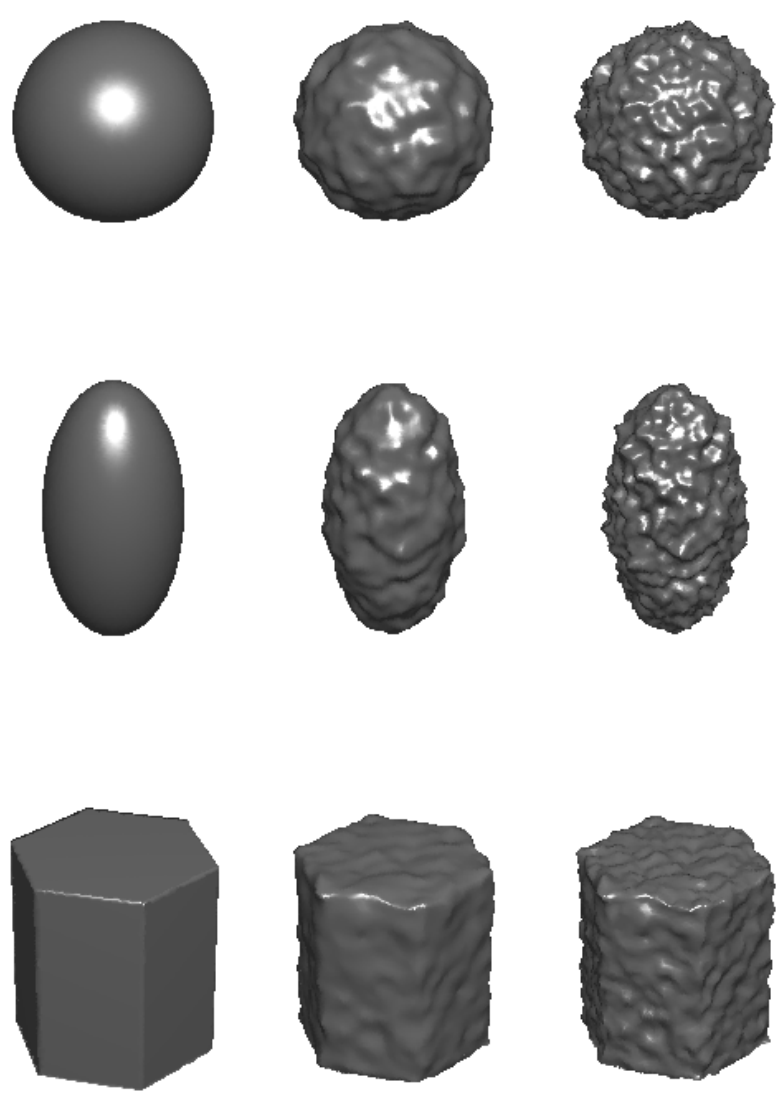

Figure 2. From left to right: particles with increasing surface height "width" $w=0.00$, $0.01,0.02$, when scaled by a (see text for definitions of $\mathrm{w}$ and a). Top row: spheres. Middle row: spheroids, polar radius/equatorial radius $=2$. Bottom row: hexagonal prisms with unit aspect ratio (drawings are not exactly to scale). 

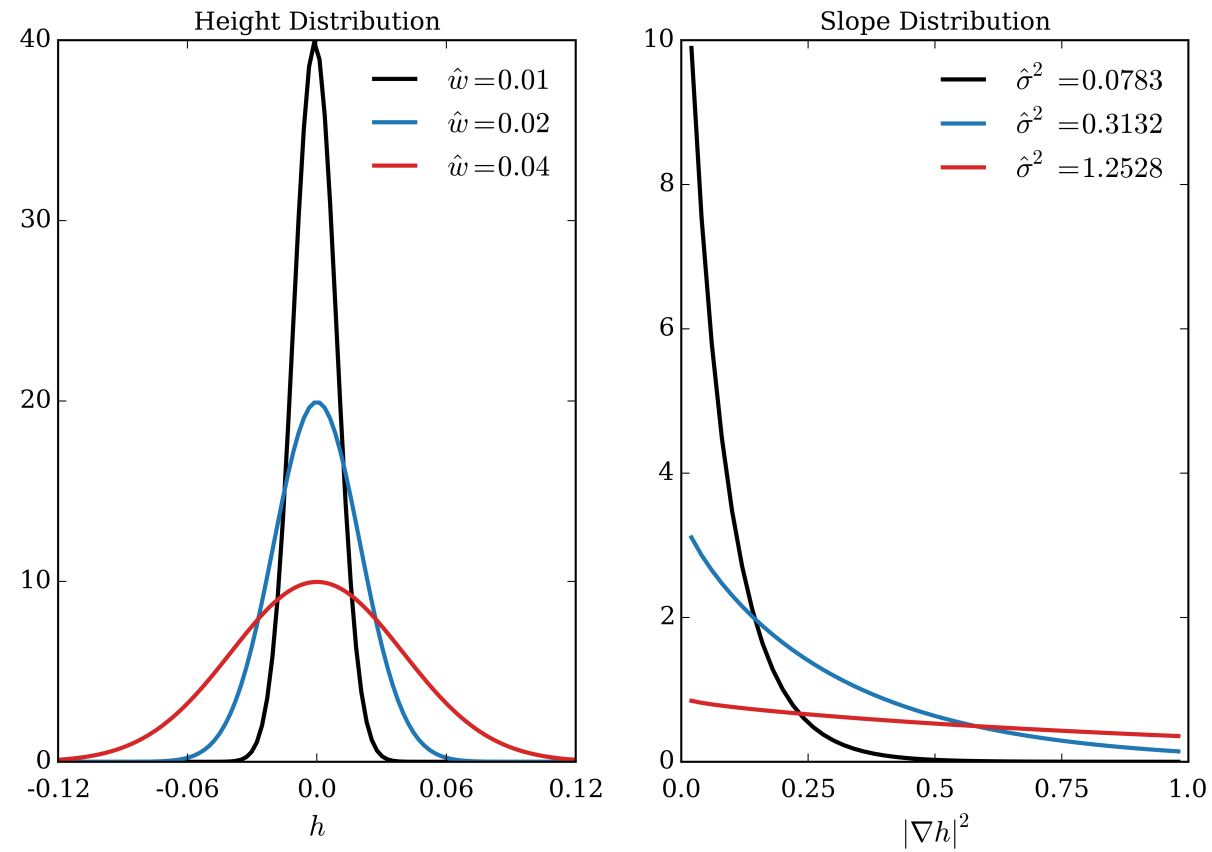

Figure 3. Estimated height and slope distributions from a roughened plane (length: 1.0, width: 0.35$)$ generated with our method. The average heights are $0.0000,0.0000$, and 0.0001 respectively. $\hat{w}$ and $\hat{\boldsymbol{\sigma}}^{2}$ are the estimated values for $\mathrm{w}$ and $\sigma^{2}$. The estimated value of $b$ is 0.9759 (Gaussian distribution: $b=1$ ) for all three Weibull distributions. 


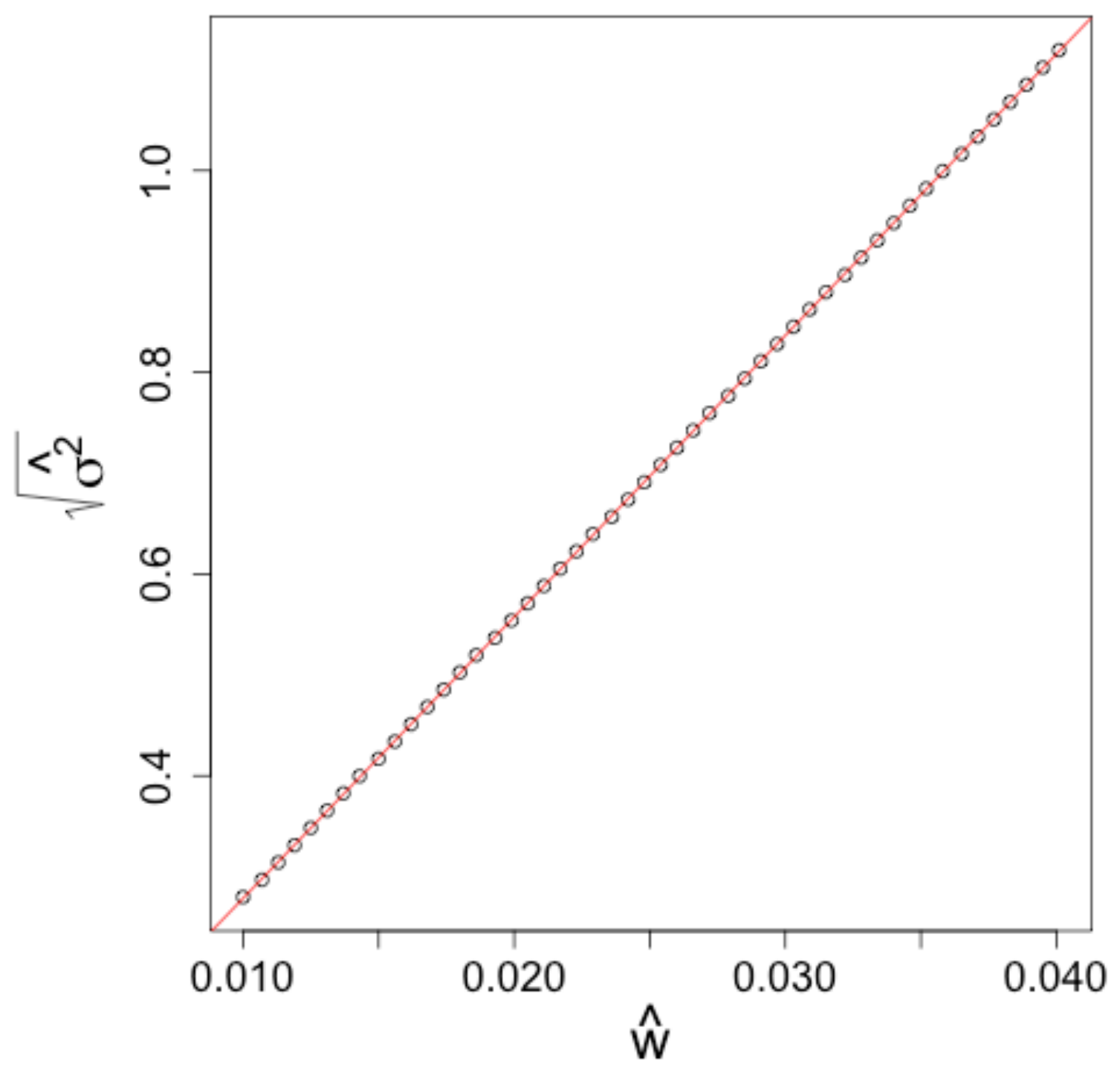

Figure 4. Plot of $\sqrt{\hat{\sigma}^{2}}$ against $\hat{w}$. The regression line fitted to the data set has $\sqrt{\hat{\sigma}^{2}}=27.90 \hat{w}-0.0004$. 

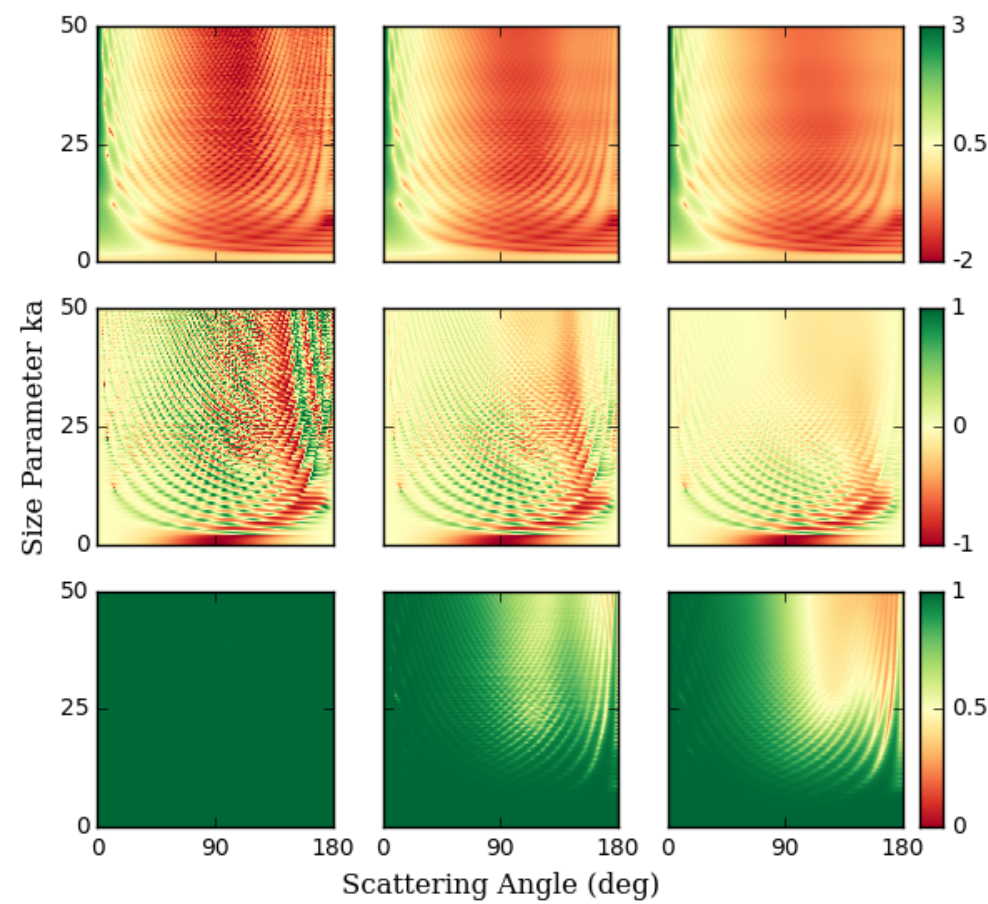

Figure 5: Phase matrix ensembles (from top row to bottom row: $\mathrm{P}_{11},-\mathrm{P}_{12} / \mathrm{P}_{11}, \mathrm{P}_{22} / \mathrm{P}_{11}$ ) computed by II-TM for roughened spheres with different height width (from left column to right column: $0.00,0.01,0.02)$. 

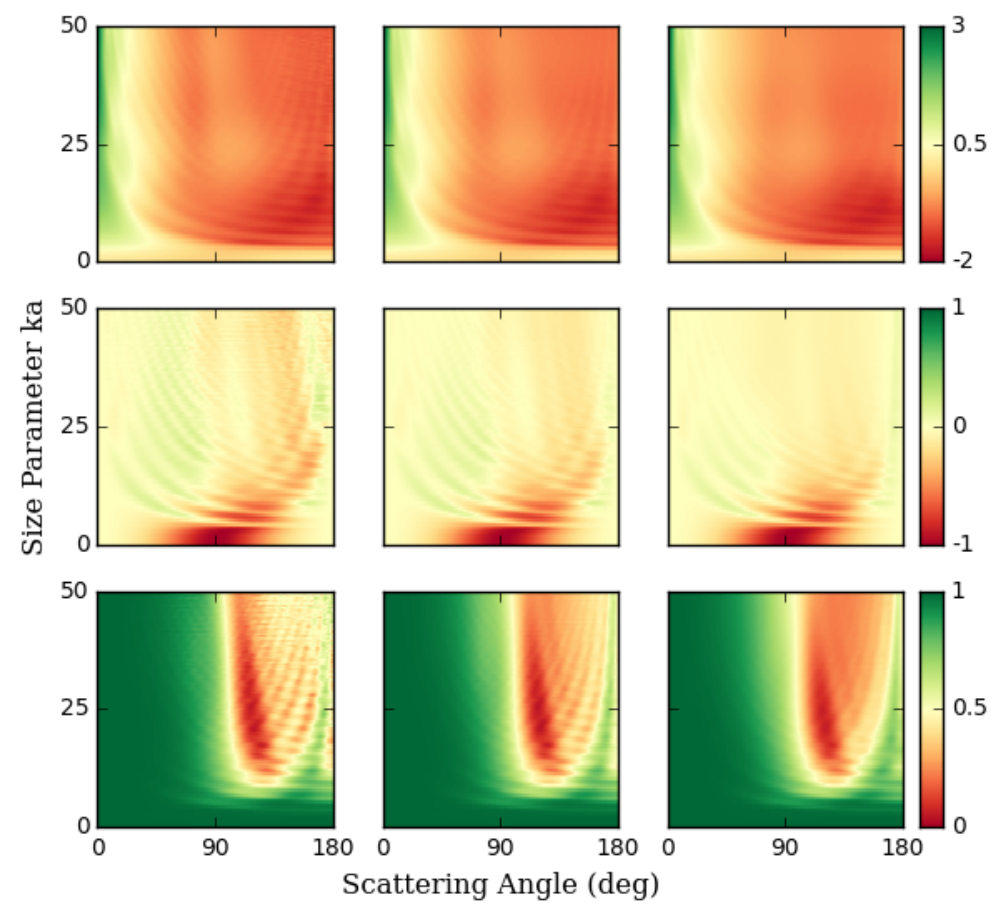

Figure 6: Phase matrix ensembles (from top row to bottom row: $\mathrm{P}_{11},-\mathrm{P}_{12} / \mathrm{P}_{11}, \mathrm{P}_{22} / \mathrm{P}_{11}$ ) computed by IITM for roughened spheroids with different height width (from left column to right column: $0.00,0.01,0.02)$. 

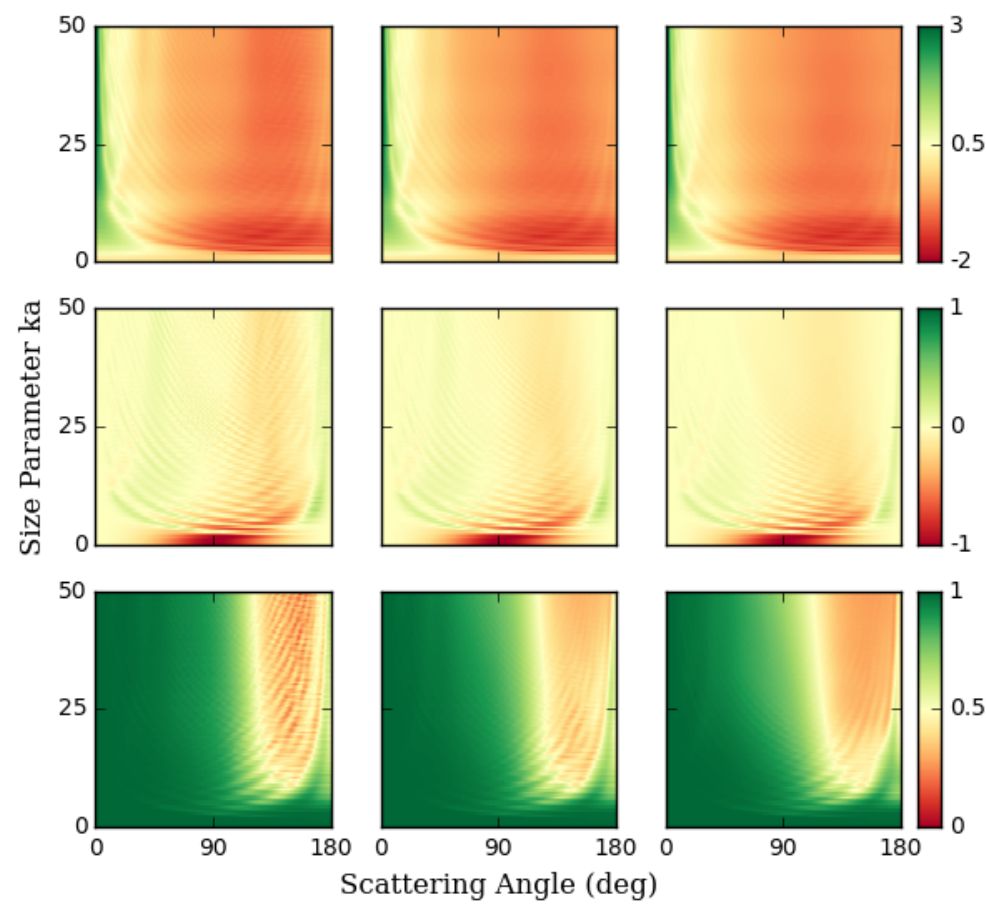

Figure 7: Phase matrix ensembles (from top row to bottom row: $\mathrm{P}_{11},-\mathrm{P}_{12} / \mathrm{P}_{11}, \mathrm{P}_{22} / \mathrm{P}_{11}$ ) computed by II-TM for roughened hexagonal prisms with different height width (from left column to right column: $0.00,0.01,0.02$ ). 

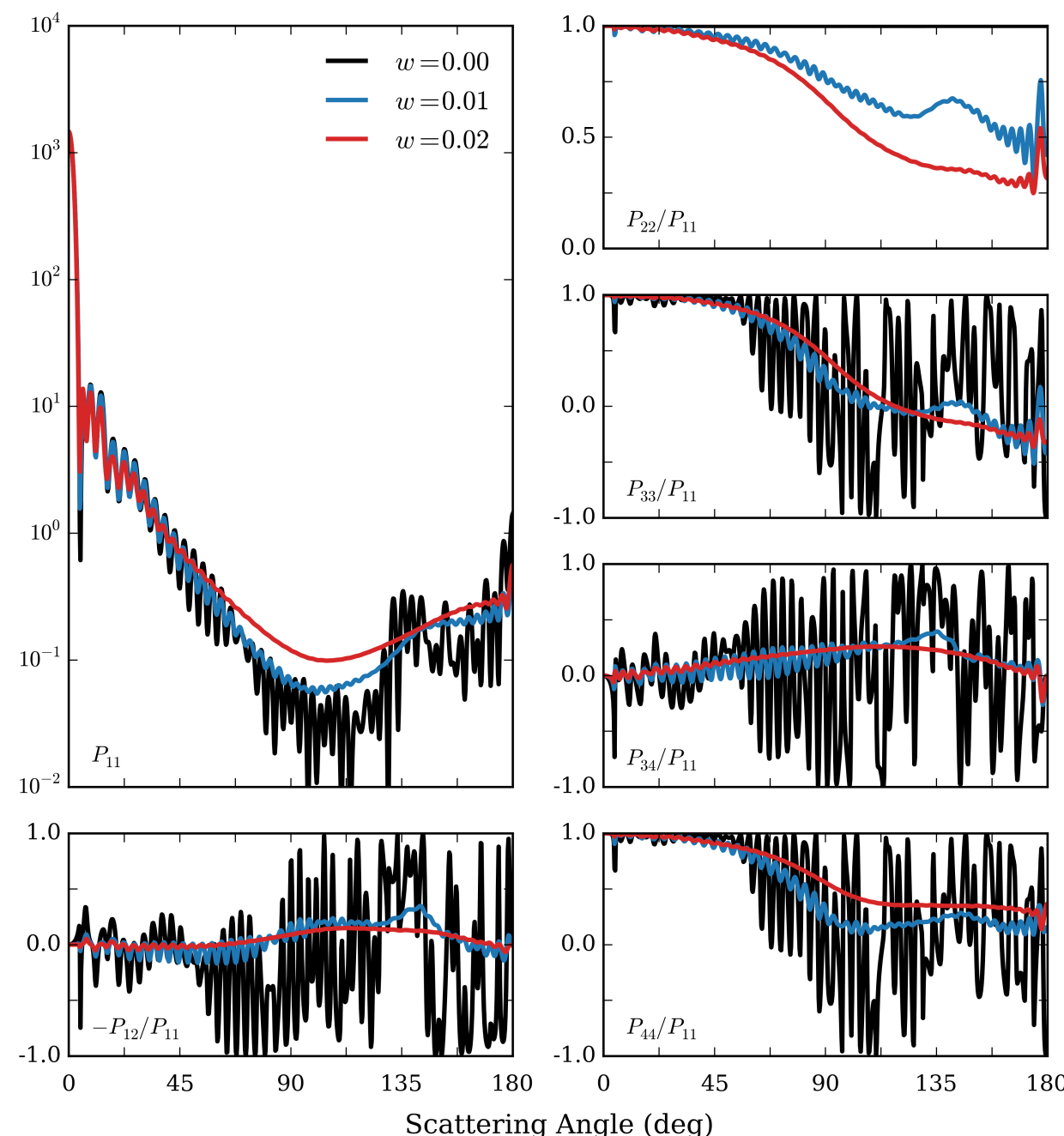

Figure 8: Phase matrix elements computed by II-TM for spheres $(\mathrm{ka}=50)$ using height distributions with different values of $\mathrm{w}$. 

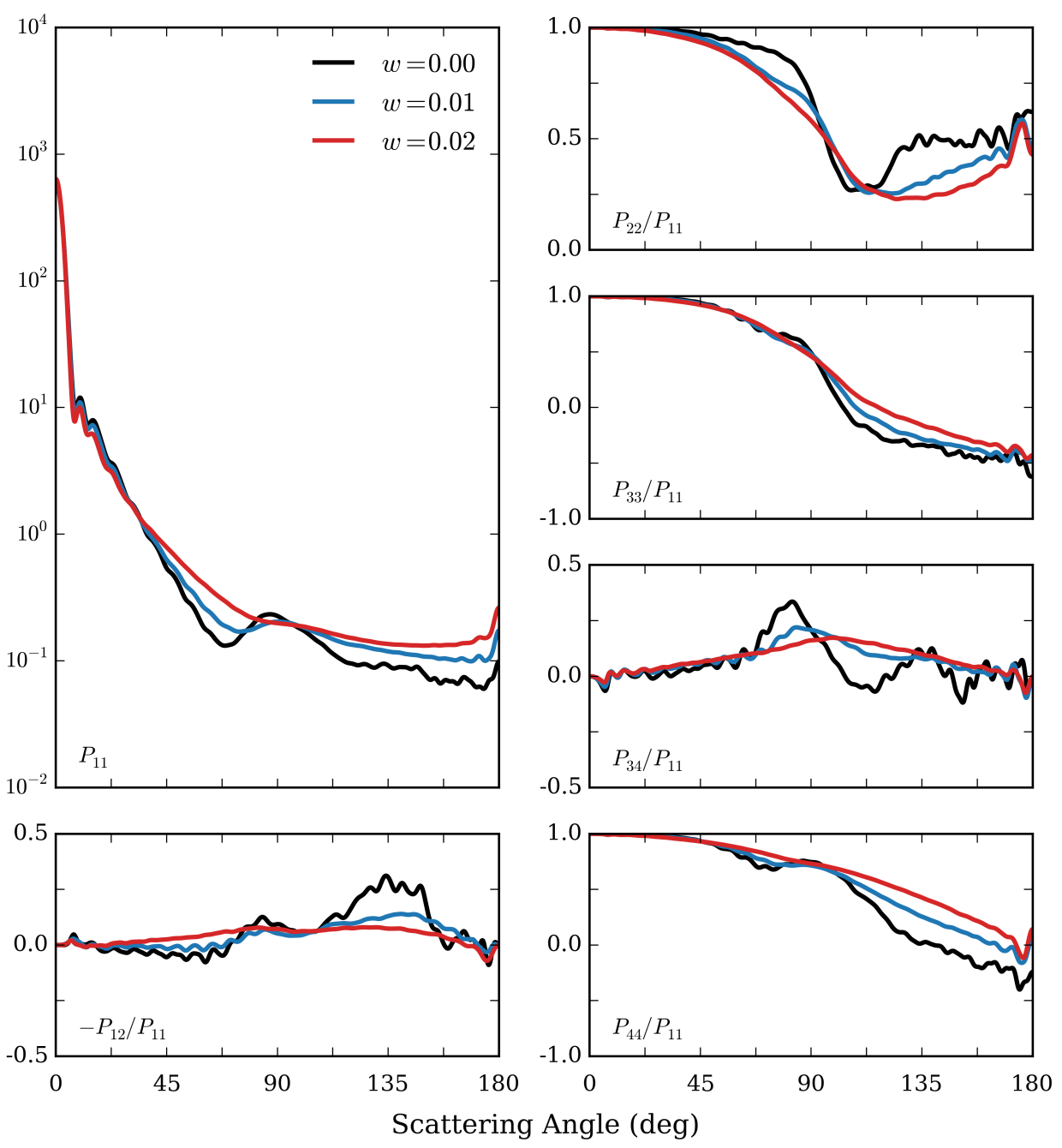

Figure 9. Phase matrix elements computed by II-TM for spheroids $(\mathrm{ka}=50)$ with different $w$. 

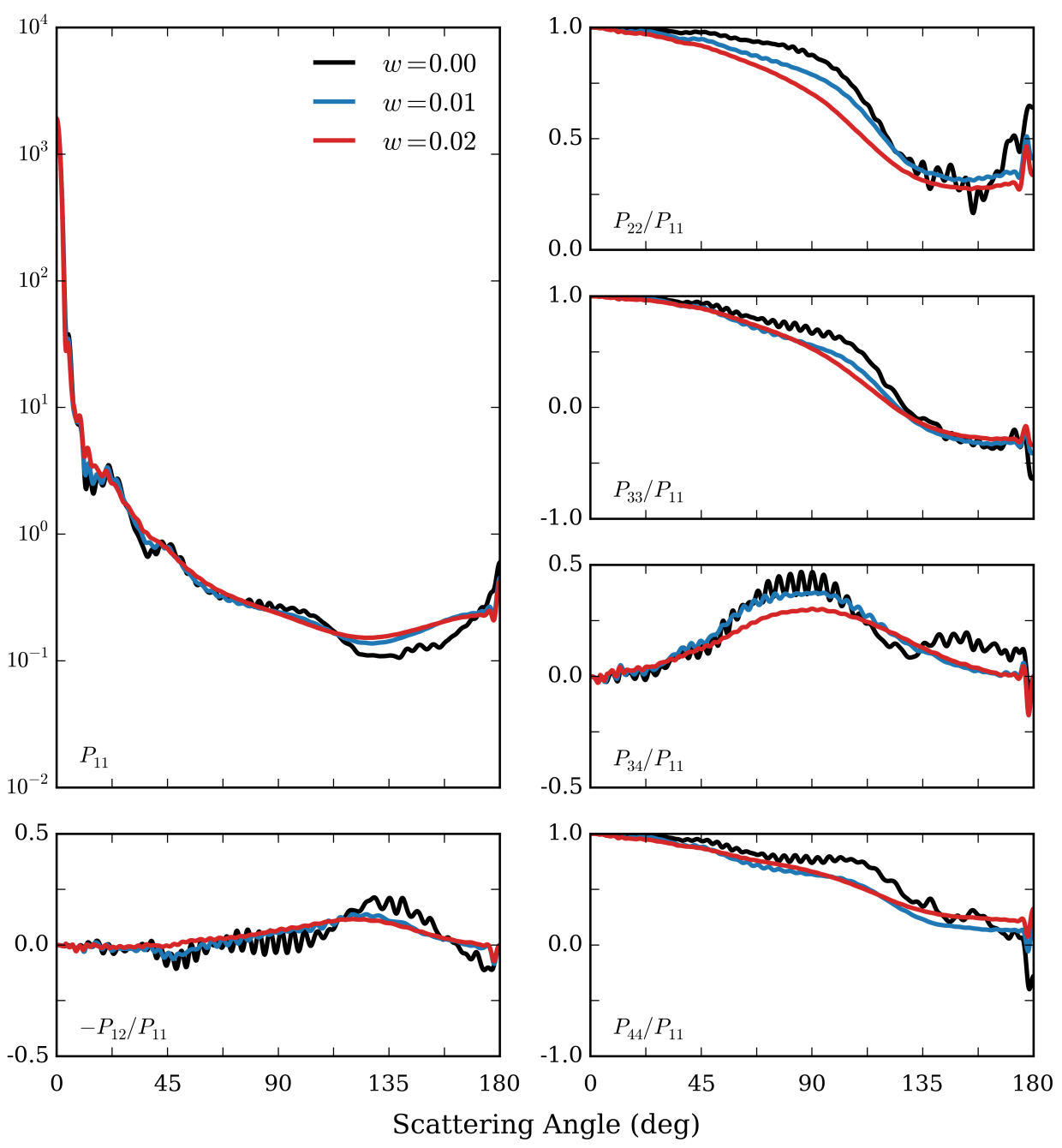

Figure 10: Phase matrix computed by II-TM for hexagonal prisms $(\mathrm{ka}=50)$ with different $\mathrm{w}$. 

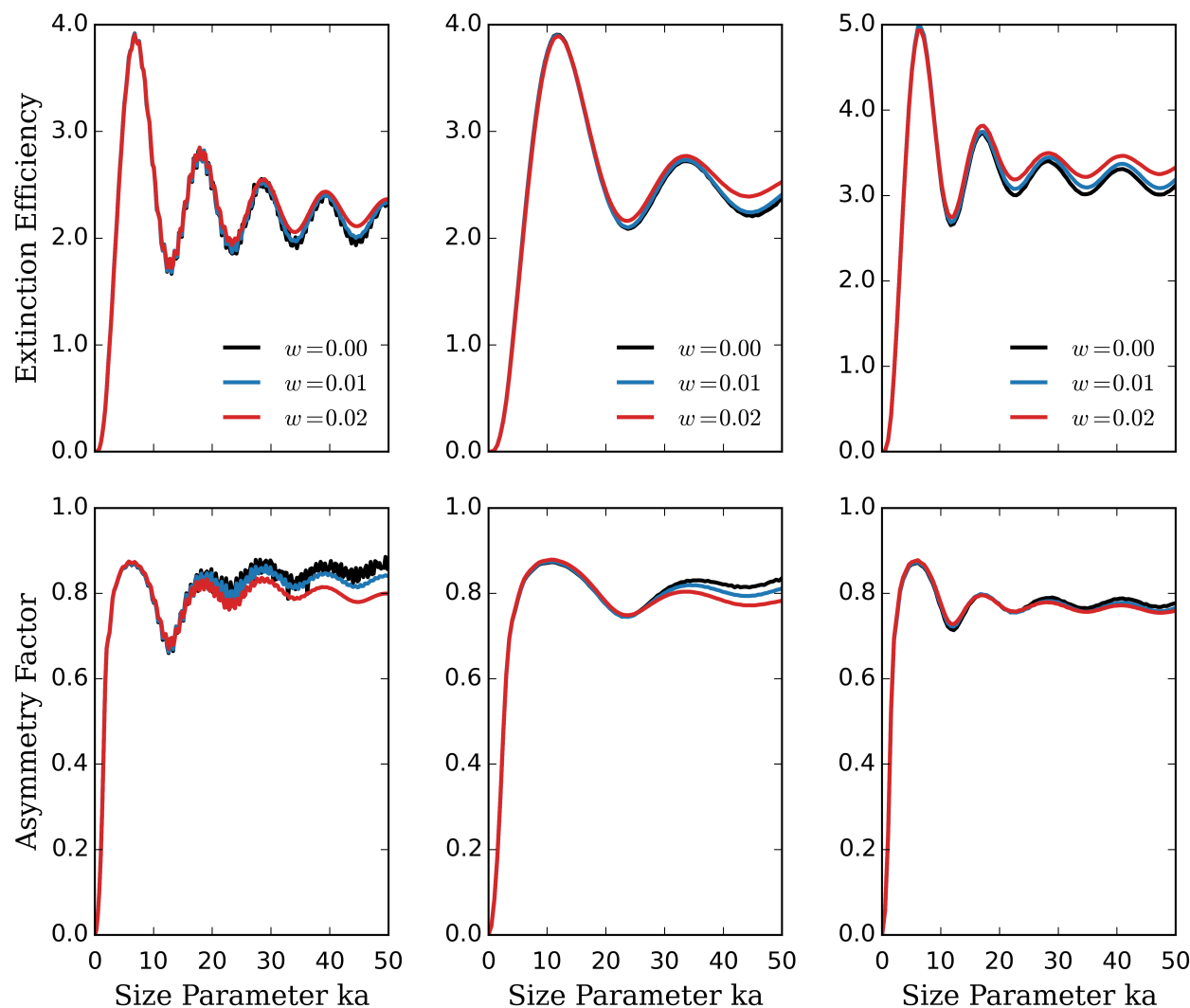

Figure 11. Extinction efficiency and asymmetry factor for spheres (left), spheroids (middle), and hexagonal prisms (right) as a function of size parameter. 